\title{
Early changes in diaphragmatic function evaluated using ultrasound in cardiac surgery patients: a cohort study
}

\author{
António Tralhão ${ }^{1,5} \cdot$ Pedro Cavaleiro $^{1,6} \cdot$ Mattia Arrigo $^{1,7}$. Jean-Paul Lopes ${ }^{1} \cdot$ Marion Lebrun $^{1}$. \\ Mercedes Rivas-Lasarte ${ }^{1,8}$. Françoise Le Pimpec-Barthes ${ }^{2,4}$. Christian Latrémouille ${ }^{3,4}$. Paul Achouh ${ }^{3,4}$. \\ Romain Pirracchio ${ }^{1,4} \cdot$ Bernard Cholley ${ }^{1,4}$
}

Received: 25 March 2019 / Accepted: 1 July 2019 / Published online: 5 July 2019

(c) Springer Nature B.V. 2019

\begin{abstract}
Little is known about the evolution of diaphragmatic function in the early post-cardiac surgery period. The main purpose of this work is to describe its evolution using ultrasound measurements of muscular excursion and thickening fraction (TF). Single-center prospective study of 79 consecutive uncomplicated elective cardiac surgery patients, using motion-mode during quiet unassisted breathing. Excursion and TF were measured sequentially for each patient [pre-operative (D1), 1 day (D2) and 5 days (D3) after surgery]. Pre-operative median for right and left hemidiaphragmatic excursions were 1.8 (IQR 1.6 to $2.1) \mathrm{cm}$ and $1.7(1.4$ to 2.0$) \mathrm{cm}$, respectively. Pre-operative median right and left thickening fractions were 28 (19 to 36) \% and 33 (22 to 51) \%, respectively. At D2, there was a reduction in both excursion (right: 1.5 (1.1 to 1.8) cm, p < 0.001, left: 1.5 (1.1 to 1.8$), p=0.003$ ) and thickening fractions (right: 20 (15 to 34) $\%, p=0.021$, left: 24 (17 to 39) \%,p=0.002), followed by a return to pre-operative values at $\mathrm{D} 3$. A positive moderate correlation was found between excursion and thickening fraction (Spearman's rho 0.518 for right and 0.548 for left hemidiaphragm, $\mathrm{p}<0.001$ ). Interobserver reliability yielded a bias below $0.1 \mathrm{~cm}$ with limits of agreement (LOA) of $\pm 0.3 \mathrm{~cm}$ for excursion and $-2 \%$ with LOA of $\pm 21 \%$ for thickening fractions. After cardiac surgery, the evolution of diaphragmatic function is characterized by a transient impairment followed by a quick recovery. Although ultrasound diaphragmatic excursion and thickening fraction are correlated, excursion seems to be a more feasible and reproducible method in this population.
\end{abstract}

Keywords Diaphragm $\cdot$ Cardiac surgery $\cdot$ Ultrasound $\cdot$ Excursion, thickening

António Tralhão and Pedro Cavaleiro have equally contributed to this work.

Electronic supplementary material The online version of this article (https://doi.org/10.1007/s10877-019-00350-8) contains supplementary material, which is available to authorized users.

António Tralhão

atralhao@gmail.com

1 Department of Anesthesia and Intensive Care, Hôpital Européen Georges Pompidou, AP-HP, Paris, France

2 Department of Thoracic Surgery, Hôpital Européen Georges Pompidou, AP-HP, Paris, France

3 Department of Cardiac Surgery, Hôpital Européen Georges Pompidou, AP-HP, Paris, France

4 Université Sorbonne-Paris-Cité, Paris, France

5 Present Address: Department of Cardiology, Hospital de Santa Cruz, Centro Hospitalar de Lisboa

\author{
List of abbreviations \\ CABG Coronary artery bypass grafting \\ COPD Chronic obstructive pulmonary disease \\ cm Centimeter(s) \\ DE Diaphragmatic excursion \\ h Hours
}

Ocidental, Av. Professor Doutor Reinaldo dos Santos, 2790-134 Carnaxide, Portugal

6 Present Address: Intensive Care Department, Centro Hospitalar Universitário do Algarve, Hospital de Faro, Faro, Portugal

7 Present Address: Department of Cardiology, University Heart Center, University Hospital Zurich, Zurich, Switzerland

8 Present Address: Cardiology Department, Hospital de la Santa Creu i Sant Pau, Biomedical Research Institute IIB-Sant Pau, Universidad Autónoma de Barcelona, Barcelona, Spain 


$\begin{array}{ll}\text { LOA } & \text { Limits of agreement } \\ \text { M } & \text { Motion } \\ \text { MHz } & \text { Megahertz } \\ \text { mm } & \text { Millimeter(s) } \\ \text { TF } & \text { Thickening fraction } \\ \text { US } & \text { Ultrasound }\end{array}$

\section{Introduction}

Impaired diaphragmatic function following cardiac surgery due to mechanical or hypothermal damage to the phrenic nerve has been well described [1-4]. Although moderate in most cases, up to $6 \%$ of patients may suffer more severe forms of diaphragmatic dysfunction leading to prolonged mechanical ventilation and associated pulmonary complications [5]. But apart from clinically relevant diaphragmatic dysfunctions, little is known about the natural course of diaphragmatic function in the early post-operative period. Providing such description would help to better understand the impact of cardiac surgery on diaphragm function.

Among the different tools to assess diaphragm performance, ultrasound (US) techniques have gained an increasingly prominent role as a non-invasive bedside imaging modality due to their simplicity, non-invasiveness, feasibility and reproducibility [6-9]. US measurements have also shown good correlation with the reference method, i.e., transdiaphragmatic pressure measurement, whose semi-invasive nature, complexity and expertise requirement compare unfavorably against echography in daily clinical use [6].

Currently, US evaluation of the diaphragm is based on regional excursion and thickening measurements, mainly through a lateral thoracic or subcostal approach $[6,7]$. Although previous studies have established the range of normal values for these two indices, their feasibility and reproducibility was never compared in cardiac surgery patients $[8,10]$.

The primary aim of this study was to compare the excursion and thickening of the diaphragm before and after cardiac surgery, by analyzing bilateral diaphragmatic excursion (DE) and thickening fraction (TF) during spontaneous breathing. In addition, we compared the reproducibility and feasibility of both measurements.

\section{Materials and methods}

This was a single center study conducted prospectively at a tertiary university-affiliated hospital, between October and December 2015. All consecutive patients admitted to the Cardiac Surgical Department prior to elective cardiac surgery were screened for enrollment. Informed consent was obtained from all individual participants included in the study. Ethical approval for this study was provided by the local ethics committee (Comité d'éthique pour la recherche en Anesthésie-Réanimation, IRB 00010254).

\subsection{Patients and procedures}

Patients over 18 years of age undergoing elective cardiac surgery via sternotomy with normothermic cardiopulmonary bypass were eligible for inclusion. Exclusion criteria were: (a) hemodynamic instability or respiratory distress precluding quiet spontaneous breathing during the first 48 postoperative hours (h) (b) thoracotomy and (c) absence of informed consent. All procedures were performed under general anesthesia and cardiopulmonary bypass. Clinical variables and surgical related details such as the optional use of topical hypothermia ("slushed ice") and whether one or two internal thoracic arteries were employed during coronary bypass grafting were recorded.

\subsection{Ultrasound measurements}

Excursion and thickness of both hemidiaphragms were measured sequentially in each patient at three different time points: (D1)—pre-operatively (the day before surgery), (D2) - within the first $48 \mathrm{~h}$ after extubation and (D3)between 96 and $174 \mathrm{~h}$ after extubation. The number of hours between extubation and each exam was recorded.

All examinations were performed at bedside with the patient in a $45^{\circ}$ semi-recumbent position during quiet, unassisted breathing. A commercially available US platform (Philips CX50 CompactXtreme ${ }^{\circledR}$ ) connected to a 2.5-3.5 Megahertz (MHz) phased-array transducer (excursion) or a 3-12 MHz linear transducer (thickness) was used, with simultaneous display of electrocardiogram and thoracic impedance, to help in identifying each phase of the respiratory cycle.

DE was evaluated using anatomical motion (M)-mode through a lateral approach from the mid-axillary line as previously described $[6,11]$. Imaging was obtained and recorded using a slow scrolling display speed $[<25 \mathrm{~mm}$ $(\mathrm{mm})$ per second], allowing the analysis (from end-expiration to end-inspiration), measurement and average of 3 consecutive respiratory cycles on the same screen (Fig. 1a). Imaging quality check included regularity in amplitude and frequency of the DE and visibility of the diaphragmatic line throughout the respiratory cycle. In each patient, to ensure that all measurements were made from the same acoustic window, the distance between probe placement and the lower extremity of the ribcage on the mid-axillary line was measured and used as the reference site for the subsequent exams. Under normal quiet breathing conditions, hemidiaphragm dysfunction was defined as an excursion $<1 \mathrm{~cm}$ $(\mathrm{cm})$ downwards during inspiration, and diaphragmatic 


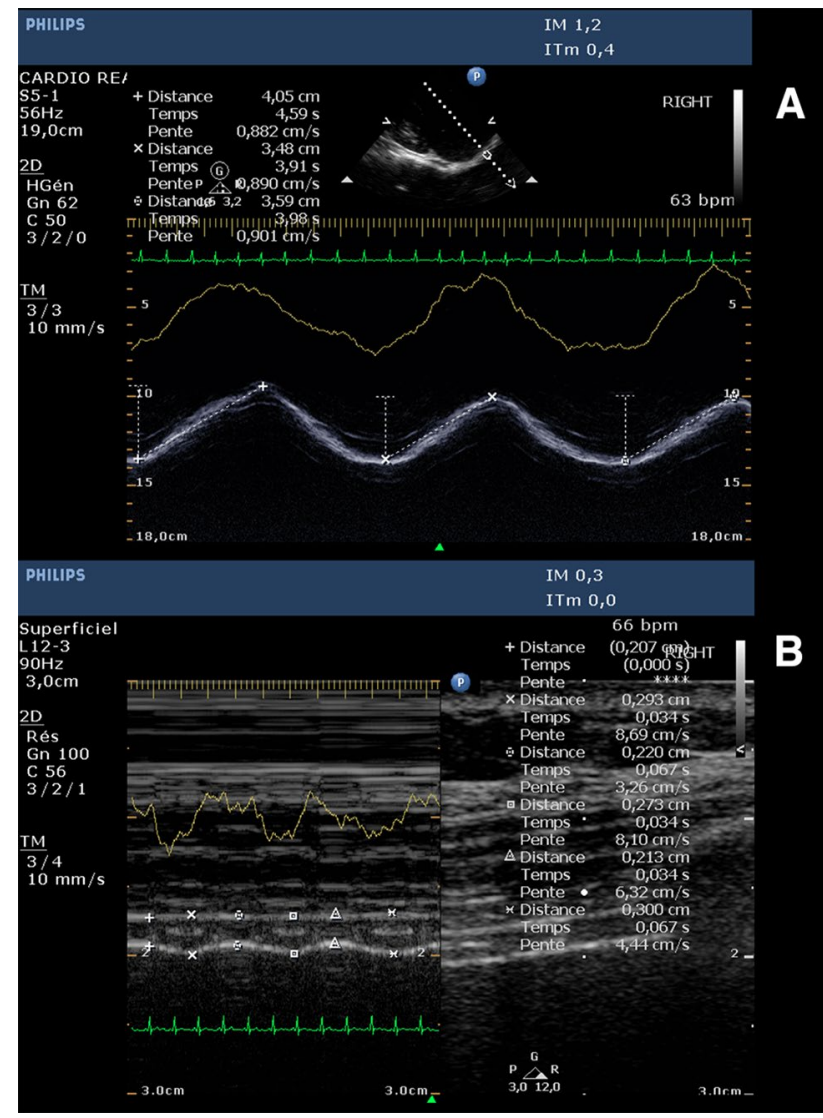

Fig. 1 a Diaphragmatic excursion obtained using anatomical M-Mode with a 2.5-3.5 MHz transducer. Measurements (dotted lines between cursors) were obtained over 3 consecutive respiratory cycles. The yellow line corresponds to variations in thoracic impedance with respiration. b Diaphragmatic thicknesses (distance between vertically placed cursors) measured using regular M-Mode with a 3-12 MHz transducer at end-expiration and end-inspiration. Measurements were obtained at end-expiration (minimal thickness) and end-inspiration (maximal thickness) over 3 consecutive respiratory cycles. The yellow line corresponds to variations in thoracic impedance with respiration

paralysis corresponded to an absence of motion $(0 \mathrm{~cm})$ or a paradoxical motion (diaphragmatic ascension during inspiration) [8].

Hemidiaphragm thickness imaging was performed at the point where the muscle fibers make contact with the rib cage, between the 8th and 10th intercostal spaces on the mid-axillary line, the so-called zone of apposition, angling the ultrasound beam close to $90^{\circ}$ [10].Correct measurement requires proper recognition of both pleural and peritoneal leaflets as continuous hyperechoic lines surrounding the structure of interest. The diaphragmatic muscle layer was then measured between the pleura and peritoneum at both end-inspiration and end-expiration using regular M-mode, and the values averaged over 3 consecutive respiratory cycles (Fig. 1b). Hemidiaphragm thickening was defined as the difference between end-inspiratory and end-expiratory thickness values, measured in $\mathrm{mm}$. We further calculated TF as:

$\mathrm{TF}=\frac{\text { Inspiratory thickness }- \text { expiratory thickness }}{\text { Expiratory thickness }} \times 100$

Exams were performed individually by experienced operators (AT, PC, MA, JPL, ML, MRL). In order to measure interoperator reliability, the first 20 patients had their examinations at D1, D2 and D3 performed twice, by two different operators (AT, PC, MA). The first operator selected the best acoustic window and registered the measurement site as described above. The order of the operators and of each hemidiaphragm evaluation was randomized using a computer-generated sequence.

\subsection{Statistical analysis}

Results are reported as mean \pm standard deviation, median (IQR 25th to 75th percentiles) or number (proportion). Continuous variables were compared using Wilcoxon's signed-rank test. Comparison between repeated measures was performed using Friedman's test. Spearman correlation coefficient ( $r h o$ ) was used to evaluate the correlation between variations in diaphragmatic excursion and TF. The Chi square test, Mann-Whitney's U or $t$ test were used for univariate analysis of diaphragmatic dysfunction occurrence, as appropriate. The Bland and Altman method for repeated measurements was performed to describe the interoperator reliability for both excursion and thickness measurements [12]. A p-value smaller than 0.05 was used to define statistical significance. All analyses were performed using SPSS 22.0 (IBM, Chicago, IL) and Stata (Release 14. College Station, TX: StataCorp LP).

\section{Results}

The study flowchart is presented in Fig. 2. Patient characteristics are presented in Table 1. Median time to diaphragm evaluation was 19 (13 to 29) $\mathrm{h}$ after extubation for D2 and 120 (114 to 136) $\mathrm{h}$ after extubation for D3.

\subsection{Diaphragmatic excursion}

DE measurements were obtained in all 79 patients for D1, 67 patients for D2 and 70 patients for D3. 67 patients (85\%) had measurements obtained at all the 3 pre-specified time points. Median baseline excursion at D1 was not significantly different between the right and the left hemidiaphragms (1.8 (1.6 to 2.1$) \mathrm{cm}$ versus 1.7 (1.4 to 2.0$) \mathrm{cm}, \mathrm{p}=0.06$ ). A significant time-variation of DE (Fig. 3) was found for both right and left hemidiaphragms $(p<0.001$ for right, $p=0.032$ for left 


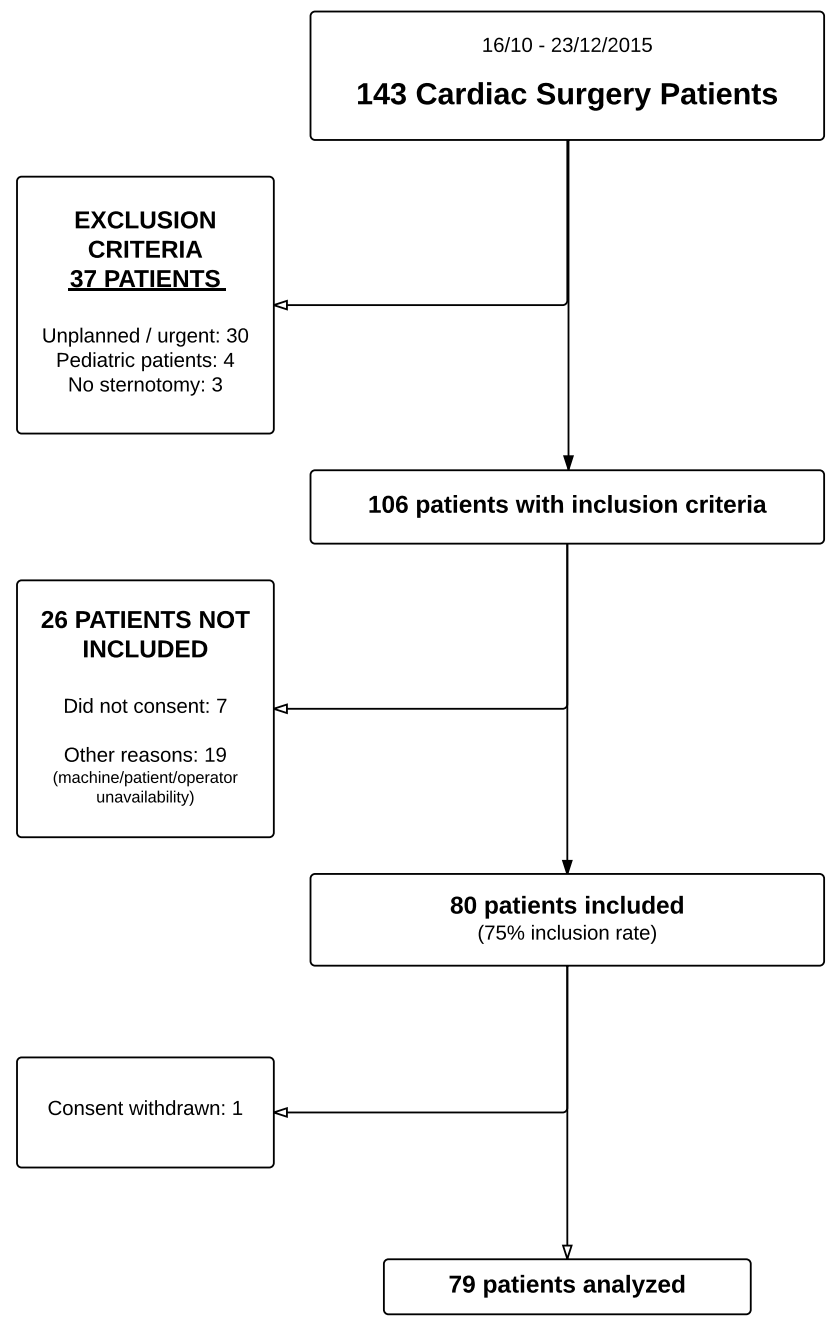

Fig. 2 Study flowchart

hemidiaphragm, Friedman's test). Using D1 as the reference, a shorter excursion was observed on D2, for right (1.5 (1.1 to 1.8$) \mathrm{cm}, \mathrm{p}<0.001)$ and left hemidiaphragms (1.5 (1.1 to $1.8), \mathrm{p}=0.003)$, followed by a return to near-baseline values on D3 (1.8 (1.4 to 2.0) $\mathrm{cm}, \mathrm{p}=0.204$ for the right; 1.6 (1.4 to 2.1$) \mathrm{cm}, \mathrm{p}=0.323$ for the left hemidiaphragm) vs. D1. Median reduction in DE measured at D2 was 0.5 (0.0 to $0.8) \mathrm{cm}$ and $0.2(-0.2$ to 0.8$) \mathrm{cm}$ for right and left hemidiaphragms, respectively.

\subsection{Hemidiaphragmatic dysfunction or paralysis}

Considering the threshold value of $1.0 \mathrm{~cm}$, diaphragmatic dysfunction was present in $25(36 \%)$ patients at D2-4 patients with bilateral dysfunction and 21 patients with unilateral dysfunction (12 right-sided, 9 left-sided), which persisted on D3 in 8 (12\%) patients-all unilateral (1 rightsided, 8 left-sided). New onset of diaphragmatic dysfunction after D2 was observed in $1(1 \%)$ patient. New onset unilateral diaphragmatic paralysis was noted in $4(5.8 \%)$ patients at $\mathrm{D} 2$ (all persistent) and in $1(1 \%)$ additional patient at D3.

\subsection{Diaphragmatic thickening fraction}

Diaphragmatic thickness measurements were obtained in 57 patients at D1 and 42 patients at D2 and D3 (no measure in 10 patients due to US machine unavailability and in 27 patients due to poor acoustic window). 42 patients (53\%) had measurements obtained at all 3 pre-specified time points. Median right and left hemidiaphragm thickness was 1.8 (1.6 to 2.0$) \mathrm{mm}$ and 1.8 (1.5 to 2.1$) \mathrm{mm}$ at $\mathrm{D} 1$, respectively. Median TF at baseline was significantly different between the right and left hemidiaphragms (28 (19 to 36) \% versus. 33 (22 to 51$) \%, p=0.015$ ).

Similarly to excursion, we found a significant time-variation of TF after surgery ( $p=0.039$ for right, $p=0.046$ for left hemidiaphragm, Fig. 4). Using D1 as the reference, a smaller TF was observed at D2 (20 (15 to 34) \%, p $=0.021$ for the right, and 24 (17 to 39) \%,p=0.002 for the left hemidiaphragm). This was again followed by an increase to near pre-operative levels at D3 (29 (18 to 37) \%,p $=0.84$ for the right, and 29 (19 to 50) \%, p=0.20 for the left hemidiaphragm) vs. D1.Correlation between excursion and TF

Considering D1 values as the reference, a positive moderate monotonic correlation was found between measurements in both hemidiaphragms (Spearman's rho 0.518 for the right; Spearman's rho 0.548 for the left hemidiaphragm, both $\mathrm{p}$ values $<0.001$, Supplementary Figs. S1 and S2). When D2 values were used as a reference, a similar result was found from D2 to D3, but with a weaker correlation (Spearman's rho $0.293, \mathrm{p}=0.046$ for the right; Spearman's rho 0.384, $\mathrm{p}$ value $=0.036$ for the left hemidiaphragm, Supplementary Figs. S3 and S4).

\subsection{Interobserver reliability}

Interobserver reliability was analyzed using a Bland and Altman representation on 54 pairs of measurements for excursion (Fig. 5), which yielded a bias below $0.1 \mathrm{~cm}$ with limits of agreement (LOA) of $\pm 0.3 \mathrm{~cm}$. For TF ( 37 pairs of measurements), we obtained a bias of $-2 \%$ with LOA of $\pm 21 \%$ (Fig. 6).

\subsection{Subgroup analysis}

We analyzed the incidence of diaphragmatic dysfunction according to different variables such as age, gender, coronary artery bypass grafting (CABG) versus other types of surgery, use of two internal thoracic arteries versus one in CABG patients and use of topical hypothermia versus no hypothermia. None of these variables had a statistically 
Table 1 Patient characteristics $(\mathrm{n}=79)$

\begin{tabular}{ll}
\hline Age (years) [mean (SD)] & $62(14)$ \\
Male gender & $54(68)$ \\
Weight $(\mathrm{kg})($ median $[25$ th-75th percentiles]) & $74[62-83]$ \\
Height $(\mathrm{m})[$ mean $(\mathrm{SD})]$ & $1.7(0.1)$ \\
Body mass index $\left(\mathrm{kg} / \mathrm{m}^{2}\right)$ [mean (SD)] & $26(4.7)$ \\
Body surface area $\left(\mathrm{m}^{2}\right)[$ mean (SD)] & $1.9(0.3)$ \\
COPD & \\
Type of surgery & $4(5)$ \\
Valvular & $36(42)$ \\
Coronary & $25(32)$ \\
Aortic & $2(3)$ \\
Combined & $13(17)$ \\
Other & $3(2)$ \\
Internal thoracic artery use in CABG (isolated or combined) & \\
One & $31(63)$ \\
Two & $18(37)$ \\
Cardiopulmonary bypass time (min) [mean (SD)] & $107(53)$ \\
Aortic cross clamp time (min) [mean (SD)] & $74(43)$ \\
Logistic EUROSCORE I (\%) (median [25th-75th percentiles]) & $3.5[2.0-6.2]$ \\
ASA score & \\
2 & $16(20)$ \\
3 & $57(72)$ \\
4 & $6(8)$ \\
Ventilation duration (h) (median [25th-75th percentiles]) & $11[8-18]$ \\
Use of catecholamines & $22(28)$ \\
Renal replacement therapy & $1(1)$ \\
NIPV after extubation & $2(3)$ \\
\hline & $4(5)$ \\
\hline
\end{tabular}

Values are presented as number (percentage), unless otherwise indicated

ASA American Society of Anesthesiologists; NIPV non-invasive positive-pressure ventilation, SD standard deviation, $C O P D$ chronic obstructive pulmonary disease significant association with the occurrence of post-operative diaphragmatic dysfunction (Supplementary Table S1).

\section{Discussion}

In a population of consecutive, elective cardiac surgery patients undergoing planned sternotomy, we have been able to describe the time course of diaphragmatic function during spontaneous breathing by comparing it with the preoperative state. In this regard, our results can be summarized as follows: (1) immediate post-operative diaphragmatic function is almost always reduced, (2) the nature of diaphragmatic dysfunction is transient in the majority of cases and shows a recovery by the 5 th post-operative day, (3) diaphragmatic US assessment is both feasible and reproducible but (4) diaphragm excursion seems to be superior to thickening for bedside assessment.

Diaphragmatic dysfunction is clinically relevant in cardiac surgery patients owing to a potentially negative impact on time to ventilator weaning, extubation success and intensive care unit length of stay [1-3]. Hitherto, there have been several studies analyzing the incidence and predictors of diaphragmatic dysfunction in this population using a variety of methods, but only a few have used US techniques [4-6]. As for any variable of interest, it is crucial to define normality, as this forms the basis for a correct diagnosis and prognosis. Boussuges et al. have previously set the DE threshold to define diaphragmatic dysfunction [8]. However, the dynamic characterization of diaphragm mechanics after cardiac surgery has not been described yet.

We observed a consistent pattern of global diaphragm excursion reduction [median $-0.4(-0.8$ to 0.1$) \mathrm{cm}$ ] $48 \mathrm{~h}$ after surgery. After this apparent diaphragm "stunning", a rapid normalization to pre-operative values for right and left excursion ensued. In $36 \%$ of these patients, the reduction in DE was seemingly severe enough to be graded as "dysfunction" according to current criteria [8]. Nevertheless, this greater reduction in excursion was also transient and showed rapid recovery by D3. Based on a clinical 


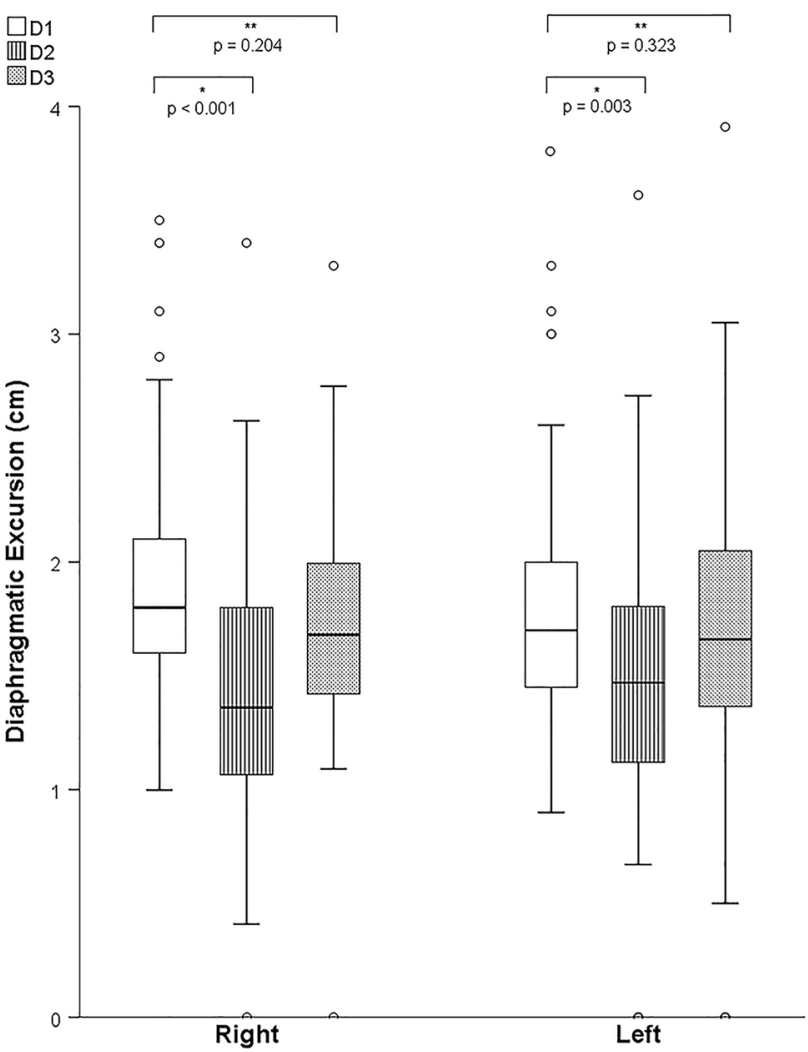

Fig. 3 Time variation of diaphragmatic excursion for right and left hemidiaphragms

uneventful course for our patient population, such dysfunction is probably benign in nature. Moreover, this alteration in diaphragmatic function, even in case of unilateral diaphragmatic paralysis, was not associated with clinical signs of respiratory impairment.

Studies on diaphragmatic function focusing on cardiac surgery patients with an uneventful course are scarce. Lerolle et al. studied the diaphragm of patients with difficult weaning from mechanical ventilation and a comparator group of patients extubated within $12 \mathrm{~h}$ postoperatively [6]. Among patients from the latter group, a reduction in DE was also encountered on the 2 nd or 3 rd post-operative day [6]. However, some differences with respect to the present study should be noted. Excursion measurements were made between forced expiration and maximal inspiration requiring patient's cooperation, whereas we studied patients breathing normally, without effort. This accounts for the difference between a $-40 \%$ reduction in diaphragmatic excursion decrease observed in the study by Lerolle et al. [6] and the one found in this study $(-17 \%)$. We chose to avoid forced inspiration because the ability to mobilize a vital capacity in the postoperative period is impaired by postoperative pain and is highly variable among patients. This also allowed us to

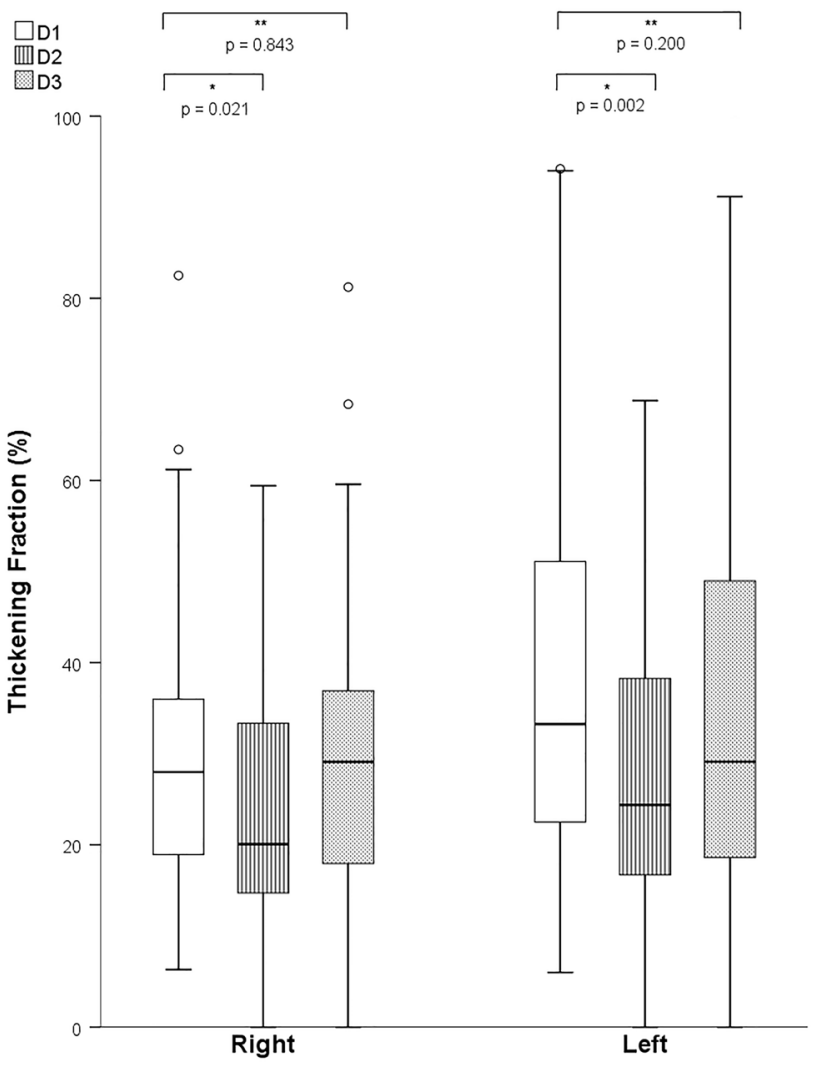

Fig. 4 Time variation of diaphragmatic thickening fraction for right and left hemidiaphragms

obtain our values by averaging 3 consecutive respiratory cycles, thereby avoiding selection bias.

In our analysis, we found a similar dynamic profile between muscular excursion and thickening. Diaphragmatic thickening during inspiration has the theoretical advantage of better reflecting the active contraction of muscle bundles [13]. While this may hold true in mechanically ventilated patients [13], such difference was not apparent from our study. Moreover, since obtaining appropriate acoustic windows for thickening imaging can be quite difficult and sometimes even impossible (34\% failure rate in our cohort), excursion appears as an easier measurement to assess the function of the diaphragm in spontaneously breathing patients. To further compare both methodologies, we undertook a reproducibility sub-study, evaluating excursion and thickening measured twice in the same patients by two different operators. Visual analysis and numerical comparison show that both methods are reproducible, although they seem to suggest that excursion is a more reproducible metric of diaphragm function than thickening. Previous authors found a good reproducibility for each parameter when measured individually $[8,10]$.The small disparity we encountered may be a reflection of the difference between the magnitude of excursion (centimeters) and thickening 
Fig. 5 Bland-Altman plot: representation of the agreement between paired diaphragmatic excursion measurements obtained by two different operators

Fig. 6 Bland-Altman plot: representation of the agreement between paired diaphragmatic thickening fraction measurements obtained by two different operators
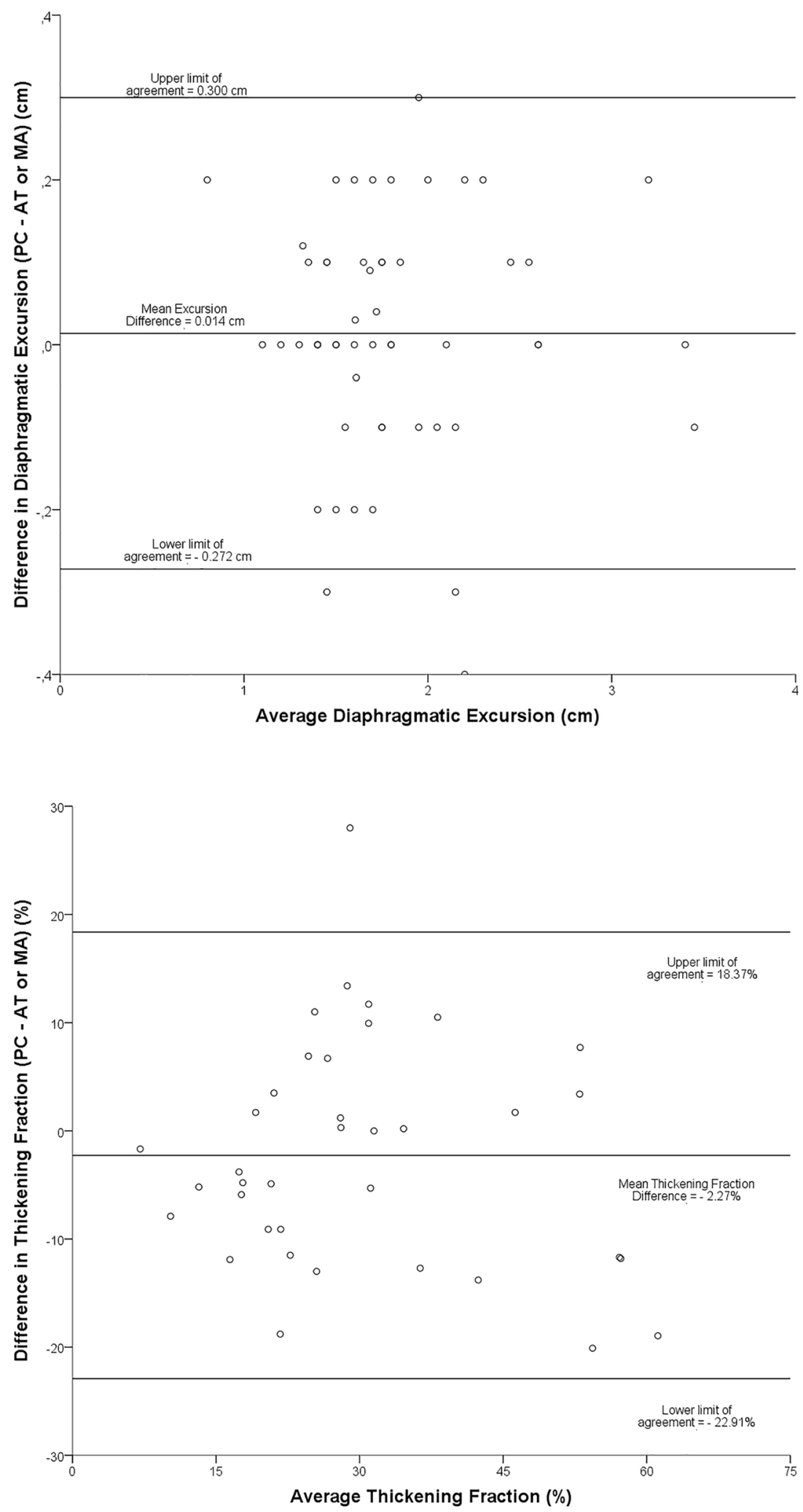

(millimeters) measurements. Due to the very small values of diaphragm thicknesses, any inaccuracy in the placement of the cursors can result in proportionately greater errors in the percentage of thickening. Finally, the correlation of diaphragmatic thickness and excursion was moderate. This finding may be interpreted as another reason to use excursion 
instead of thickening to assess diaphragmatic function due to the inherent measurement errors with the latter.

Our study has a number of strengths. First, and to the best of our knowledge, this is the first study to describe the natural course of diaphragmatic function in consecutive elective cardiac surgery patients using US as the assessment tool. Second, we have described how the diaphragm behaves after planned sternotomy using serial measurements, demonstrating a transient postoperative dysfunction of the main respiratory muscle, even in asymptomatic patients. Third, we have also added to the work of previous authors by demonstrating that such evaluation is both feasible and reproducible in this particular subset of patients.

Some limitations have to be acknowledged. This was a relatively small cohort, taking place at a single center. Due to unpredictable and uncontrollable external factors, we were unable to collect data from a small number of patients during the 3-month study-period. While we recognize the potential introduction of bias related to missing data, its influence in a study that was not designed to be statistically powered to detect meaningful differences between groups is probably negligible. We did not quantitatively assess the influence of pain (e.g. visual assessment score, analgesic dose) during image acquisition, although any meaningful impact was probably minimized by assuring that all exams were performed during quiet, unassisted breathing. Furthermore, owing to the small size of our population, interpreting the lack of association between variables such as topical hypothermia or COPD and diaphragmatic dysfunction should be made with caution. Finally, we only provide information on patients without respiratory impairment, and therefore cannot extrapolate on the role of diaphragmatic dysfunction in patients with respiratory distress or difficult weaning from mechanical ventilation following cardiac surgery, similarly to what has been proposed by some authors in different clinical settings [14].

\section{Conclusion}

In conclusion, diaphragmatic function assessed using ultrasound in spontaneously breathing patients after uncomplicated cardiac surgery is characterized by a transient reduction, followed by a swift return to preoperative values. Diaphragm excursion and thickening showed overlapping temporal profiles, but excursion was easier to acquire and more reproducible in this population.

\section{Compliance with ethical standards}

Conflicts of interest The authors declare that they have no conflict of interest.
Research involving human participants and/or animals All procedures performed in studies involving human participants were in accordance with the ethical standards of the institutional and/or national research committee (Comité d'éthique pour la recherche en Anesthésie-Réanimation, IRB 00010254) and with the 1964 Helsinki declaration and its later amendments or comparable ethical standards.

Informed consent Informed consent was obtained from all individual participants included in the study.

\section{References}

1. McCool FD, Tzelepis GE. Dysfunction of the diaphragm. N Eng J Med. 2012;366:932-42.

2. DeVita MA, Robinson LR, Rehder J, Hattler B, Cohen C. Incidence and natural history of phrenic neuropathy occurring during open heart surgery. Chest. 1993;103(3):850-6.

3. Diehl JL, Lofaso F, Deleuze P, Similowski T, Lemaire F, Brochard L. Clinically relevant diaphragmatic dysfunction after cardiac operations. J Thorac Cardiovasc Surg. 1994;107(2):487-98.

4. Markand ON, Moorthy SS, Mahomed Y, King RD, Brown JW. Postoperative phrenic nerve palsy in patients with open-heart surgery. Ann Thorac Surg. 1985;39(1):68-73.

5. Engoren M, Buderer NF, Zacharias A. Long-term survival and health status after prolonged mechanical ventilation after cardiac surgery. Crit Care Med. 2000;28(8):2742-9.

6. Lerolle N, Guerot E, Dimassi S, Zegdi R, Faisy C, Fagon JY, et al. Ultrasonographic diagnostic criterion for severe diaphragmatic dysfunction after cardiac surgery. Chest. 2000;135(2):401-7.

7. Matamis D, Soilemezi E, Tsagourias M, Akoumianaki E, Dimassi $\mathrm{S}$, Boroli $\mathrm{F}$, et al. Sonographic evaluation of the diaphragm in critically ill patients. Technique and clinical applications. Intensive Care Med. 2013;39(5):801-10.

8. Boussuges A, Gole Y, Blanc P. Diaphragmatic motion studied by M-mode ultrasonography: methods, reproducibility, and normal values. Chest. 2009;135(2):391-400.

9. Kim WY, Suh HJ, Hong SB, Koh Y, Lim CM. Diaphragm dysfunction assessed by ultrasonography: influence on weaning from mechanical ventilation. Crit Care Med. 2011;39(12):2627-30.

10. Cohn D, Benditt JO, Eveloff S, McCool FD. Diaphragm thickening during inspiration. J Appl Physiol. 1997;83(1):291-6.

11. Pasero D, Koeltz A, Placido R, Fontes Lima M, Haun O, Rienzo M, et al. Improving ultrasonic measurement of diaphragmatic excursion after cardiac surgery using the anatomical M-mode: a randomized crossover study. Intensive Care Med. 2015;41(4):650-6.

12. Bland JM, Altman DG. Statistical methods for assessing agreement between two methods of clinical measurement. Lancet. 1986;1(8476):307-10.

13. DiNino E, Gartman EJ, Sethi JM, McCool FD. Diaphragm ultrasound as a predictor of successful extubation from mechanical ventilation. Thorax. 2014;69(5):423-7.

14. Spadaro S, Grasso S, Mauri T, Dalla Corte F, Alvisi V, Ragazzi $\mathrm{R}$, et al. Can diaphragmatic ultrasonography performed during the T-tube trial predict weaning failure? The role of diaphragmatic rapid shallow breathing index. Crit Care. 2016;20(1):305.

Publisher's Note Springer Nature remains neutral with regard to jurisdictional claims in published maps and institutional affiliations. 